OS9 (4) - 15 (OSO9W0167)

\title{
Damage Detection of Hybrid CFRP Reinforcements by DC Measurement Technique
}

\author{
Wu Zhishen, Dept. of Urban \& Civil Engineering, Ibaraki Univ., Hitachi, Japan \\ Yang Caiqian, Dept. of Urban \& Civil Engineering, Ibaraki Univ., Hitachi, Japan \\ Takatada Takahashi, Dept. of Urban \& Civil Engineering, Ibaraki Univ., Hitachi, Japan \\ Yoshihiro Tobe. Dept. of Urban \& Civil Engineering, Ibaraki Univ., Hitachi, Japan
}

Hybrid CFRP and CFRP composites are increasingly used as structural materials and reinforcements, +especially in the aircraft industries and civil engineerings, due to their light weight, high strength, high stiffness and good environmental compatibility with concrete. In-situ structural health monitoring, together with strain and stress detection, can improve the durability and safety of the composites structures. This present work first deals with the fabrication of continuous hybrid CFRP composites, in the forms of sheet and rod, strengthened with several types of fibers: high modulus HM, high strength HS, high Elasticity HE and aramid fibers, where the active materials are carbon fibers, which by virtue of their electrically conductive nature and piezoresistive effects enable the detections of the strain, deformation and fractures occurring in hybrid CFRP composites. Second, the self-health monitoring functions of such kinds of composites are also demonstrated by the measurement of electrical resistance with simple electronic equipment. The hybrid rods are also used as reinforcements for concrete beams, and the health of the concrete beams are demonstrated, too. The results reveal that low direct currents are in favoring of getting the electrical resistance stable during the measurements, and that the electrical resistance of CFRP composites with HS is higher than that of CFRP composites with HM under the same measuring conditions. For hybrid laminates, the stress vs strain curves can be identified by several significant zones corresponding to different types of damage mechanisms, such as the ruptures of carbon fibers, matrix cracks and delamination between plies. During the initial low strain zone, the fractional increase in electrical resistance changes linearly in proportion to the applied tensile stress due to the piezoresistivity of the carbon fibers, as makes it possible for the application of hybrid CFRP composites as strain sensors to monitor the strain and stress of structures in real time. During the high strain zones, the electrical resistance changes in a spepwise manner with increasing the applied strain. In addition to being able to be used as reinforcements and structural materials, the results also reveal that hybrid CFRP composites possess, intrinsically, the functions of monitoring the structural health, strain and damage together with classifying the different damage mechanisms in static loading conditions. The results also evidently demonstrate another important function of hybrid CFRP composites that the hybrid composites are able to provide a useful warning of the impending of catastrophic fractures, that is, they can sustain further loading due to some high elongation fibers in the hybrid CFRP composites even after the initial rupture of load-carrying carbon fibers. 